\title{
An analysis of the generalized TODIM method
}

\author{
Bonifacio Llamazares* \\ Departamento de Economía Aplicada, Instituto de Matemáticas (IMUVA), and BORDA Research Unit, Universidad de \\ Valladolid, Avda. Valle de Esgueva 6, 47011 Valladolid, Spain.
}

\begin{abstract}
The TODIM (an acronym in Portuguese for Interactive and Multicriteria Decision Making) method is a multicriteria procedure that is receiving increasing attention from the scientific community over the last few years. In this paper, we introduce a simplified version of this procedure, which allows us to easily show that this method is vulnerable to two paradoxes affecting the weights of the model. In order to overcome these inconsistencies, we propose a generalization of the TODIM method and establish conditions under which the previous paradoxes can be avoided. Moreover, we also show that the simple additive weighting (SAW) method and, under certain hypotheses, the PROMETHEE II method can be obtained as specific cases of the generalized TODIM method.
\end{abstract}

Keywords: Multiple criteria analysis, TODIM method, weight consistency, weight monotonicity, SAW method and PROMETHEE method.

\section{Introduction}

Multiple criteria decision making (MCDM) has experienced significant development in the last few decades due mainly to the wide variety of problems that can be addressed using this methodology. A broad survey of the current state of this field can be found in Greco et al. [20]. Nowadays, multicriteria methods are an essential tool in the resolution of many real-world decision making problems in which different criteria have to be taken into account (see, for instance, Mulliner et al. [30], Diaz-Balteiro et al. [10], and Marttunen et al. [29]).

TODIM (an acronym in Portuguese for Interactive and Multicriteria Decision Making) is a multicriteria method introduced by Gomes and Lima [14, 15]. The TODIM method, which is based on a pairwise

\footnotetext{
${ }^{*}$ Corresponding author. Tel.: +34-983-186544; fax: +34-983-423299.

Email address: boni@eco. uva.es (Bonifacio Llamazares)
} 
comparison of the alternatives, calculates for each criterion the dominance of one alternative over another by using the value function introduced by Kahneman and Tversky [25] (see also Tversky and Kahneman [35]) in the framework of the prospect theory. This value function, which shows an S-shaped growth curve, allows the behavior of the decision maker (DM) to be reflected with respect to gains and losses. Finally, the overall performance of each alternative is determined by using an additive function.

In recent years, the TODIM method has received increased attention from the scientific community (see, for instance, Passos and Gomes [31], Passos et al. [32], and Lee and Shih [26]), and it has been applied to different multicriteria problems. For example, the TODIM method has been used to evaluate residential properties available for rent in the city of Volta Redonda, Brazil (Gomes and Rangel [17]), to select the best option for the location of the natural gas reserves discovered in the Santos basin, Brazil (Gomes et al. [18]), and to rank chemical industries and Thermal Power Station Units (Soni et al. [34]). It is also worth noting that the TODIM method has been extended to deal with data represented by fuzzy sets (see, for instance, Fan et al. [11], Zhang and Xu [41], Wang et al. [37], Qin et al. [33], Ji et al. [23, 24], and Yu et al. [40]).

In this paper we carry out an analysis of the TODIM method. Firstly, we will present a simplification of the function that provides the dominance degree of each alternative over the others with respect to different criteria. Moreover, we will give some examples showing that this method is vulnerable to two paradoxes concerning the weights of the model. In order to overcome these inconsistencies, we will take the following steps:

1. In the framework of multicriteria methods, we will introduce two properties that avoid the occurrence of the above mentioned paradoxes. These properties will be called: weight consistency and weight monotonicity.

2. We will propose a generalization of the TODIM method.

3. We will give conditions under which the generalized TODIM method satisfies weight consistency and weight monotonicity.

Lastly, we will see that two well-known multicriteria methods, the simple additive weighting (SAW) method and the PROMETHEE II method (assuming for the latter certain hypotheses), can be obtained as particular cases of this new procedure.

The remainder of the paper is organized as follows: Section 2 is devoted to recall the TODIM method. In Section 3 we simplify this method and show two paradoxes affecting the weights of the model. In Section 4 we propose a generalization of the TODIM method and give conditions under which the previous 
paradoxes can be avoided. In Section 5 we show that some multicriteria methods such as the SAW method and, under certain hypotheses, the PROMETHEE II method are specific cases of the generalized TODIM method. Finally, some concluding remarks are provided in Section 6.

\section{The TODIM method}

Let $\mathcal{A}=\left\{A_{1}, \ldots, A_{n}\right\}$ be a finite set of alternatives and let $C=\left\{C_{1}, \ldots, C_{m}\right\}$ be a finite set of criteria in an MCDM problem. The sets of indices are denoted by $N$ and $M$, respectively. We suppose that the performance of all alternatives with respect to all criteria are known and we denote by $x_{i k}$ the performance value of alternative $A_{i}$ with respect to criterion $C_{k}$. Since criteria are usually expressed in different units, a normalization process is generally necessary to ensure that all the values be dimensionless. In this normalization process it is crucial to make a distinction between benefit criteria (whose values are always better when larger) and cost criteria (whose values are always better when smaller) ${ }^{1}$. We will suppose that a normalization process has been carried out, and we will denote by $z_{i k} \in[0,1]$ the normalized value of alternative $A_{i}$ with respect to criterion $C_{k}$. The matrix $\mathbf{Z}=\left(z_{i k}\right)_{n \times m}$ will be called the decision matrix (see Table 1).

Table 1: A typical decision matrix.

\begin{tabular}{ccccc}
\hline & $C_{1}$ & $C_{2}$ & $\cdots$ & $C_{m}$ \\
\hline$A_{1}$ & $z_{11}$ & $z_{12}$ & $\cdots$ & $z_{1 m}$ \\
$A_{2}$ & $z_{21}$ & $z_{22}$ & $\cdots$ & $z_{2 m}$ \\
$\cdots$ & $\cdots$ & $\cdots$ & $\cdots$ & $\cdots$ \\
$A_{n}$ & $z_{n 1}$ & $z_{n 2}$ & $\cdots$ & $z_{n m}$ \\
\hline
\end{tabular}

Each criterion $C_{k}$ has an associated weight $w_{k}>0$ which reflects the relative importance of criterion $C_{k}$. We assume that $\boldsymbol{w}=\left(w_{1}, \ldots, w_{m}\right)$ is a normalized weight vector; that is, $\sum_{l=1}^{m} w_{l}=1$. The pair $(\mathbf{Z}, \boldsymbol{w})$ will be called a decision problem on $\mathcal{A}$. Let $\mathcal{D}$ be the set of decision problems on $\mathcal{A}$. A multicriteria method is a function from $\mathcal{D}$ into $\mathcal{W}$, where $\mathcal{W}$ is the set of weak orders (complete and transitive binary relations) on $\mathcal{A}$.

\footnotetext{
${ }^{1}$ An interesting survey of the main methods used in the normalization of the values can be found in Jahan and Edwards [22].
} 
The TODIM method relies on a value function that provides the dominance degree of each alternative over the others with respect to the different criteria. As in the prospect theory of Kahneman and Tversky [25], the aim of this value function is to model the gain and loss attitude of the DM on each criterion. The Sshaped value function initially considered by Gomes and Lima [14] was based on the square root function, although later Gomes and González [13] proposed a more general function.

In algorithmic form, the implementation of the TODIM method entails the following steps (see Gomes et al. [16], Fan et al. [11] and Qin et al. [33], among others):

Step 1: For each $k \in M$, calculate the relative weight of criterion $C_{k}$ to a reference criterion $C_{r}$, that is,

$$
w_{k r}=\frac{w_{k}}{w_{r}} .
$$

The reference criterion $C_{r}$ has to be chosen by the DM, although it is normal to consider the criterion with the highest weight. In this case, $w_{r}=\max _{l \in M} w_{l}$.

Step 2: For each $i, j \in N$ and $k \in M$, calculate the dominance degree of alternative $A_{i}$ over alternative $A_{j}$ with respect to criterion $C_{k}$ through the following expression: ${ }^{2}$

$$
\Phi_{k}\left(A_{i}, A_{j}\right)= \begin{cases}\sqrt{\frac{w_{k r}\left(z_{i k}-z_{j k}\right)}{\sum_{l=1}^{m} w_{l r}},} & \text { if } z_{i k} \geq z_{j k}, \\ -\frac{1}{\theta} \sqrt{\frac{\left(\sum_{l=1}^{m} w_{l r}\right)\left(z_{j k}-z_{i k}\right)}{w_{k r}}}, & \text { if } z_{i k}<z_{j k},\end{cases}
$$

where $\theta>0$. This parameter determines the effect of the losses (i.e., when $z_{i k}<z_{j k}$ ): If $\theta>1$ the losses are attenuated while if $\theta<1$ the losses are amplified. So, this parameter allows DM to rank the alternatives according to the gains and the losses: for large values of $\theta$ the best alternatives are those that provide more gains while for small values of $\theta$ the best alternatives are those that provide small losses.

Step 3: For each $i, j \in N$, calculate the overall dominance degree of alternative $A_{i}$ over alternative $A_{j}$,

$$
\Phi\left(A_{i}, A_{j}\right)=\sum_{k=1}^{m} \Phi_{k}\left(A_{i}, A_{j}\right)
$$

\footnotetext{
${ }^{2}$ This approach is also utilized in other multicriteria methods. For instance, as we will see in Section 5, the PROMETHEE method uses a preference function over the values $x_{i k}-x_{j k}$ and $x_{j k}-x_{i k}$.
} 
Step 4: For each $i \in N$, calculate the overall performance of alternative $A_{i}$,

$$
\Phi\left(A_{i}\right)=\sum_{j=1}^{n} \Phi\left(A_{i}, A_{j}\right) .
$$

Step 5: For each $i \in N$, calculate the normalized overall performance of alternative $A_{i}$ by using the following expression:

$$
\xi\left(A_{i}\right)=\frac{\Phi\left(A_{i}\right)-\min _{j \in N} \Phi\left(A_{j}\right)}{\max _{j \in N} \Phi\left(A_{j}\right)-\min _{j \in N} \Phi\left(A_{j}\right)} .
$$

Step 6: Rank the alternatives according to the values $\xi\left(A_{i}\right)$. Hence, the weak order on $\mathcal{A}$ is defined by

$$
A_{i} \succcurlyeq A_{j} \Leftrightarrow \xi\left(A_{i}\right) \geq \xi\left(A_{j}\right)
$$

It is worth noting that the classical TODIM method has been generalized by Gomes and González [13] by introducing a more general parametric form of the function $\Phi_{k}\left(A_{i}, A_{j}\right)$. To do this, they consider the subjective value function introduced by Tversky and Kahneman [35],

$$
v(x)= \begin{cases}x^{\alpha}, & \text { if } x \geq 0, \\ -\lambda(-x)^{\beta}, & \text { if } x<0,\end{cases}
$$

where $0<\alpha, \beta<1$ are estimable coefficients determining the convexity/concavity of the function and $\lambda>1$ is called the loss aversion coefficient ${ }^{3}$, and define the new dominance degree of alternative $A_{i}$ over alternative $A_{j}$ with respect to criterion $C_{k}$ by:

$$
\Phi_{k}\left(A_{i}, A_{j}\right)= \begin{cases}\left(\frac{w_{k r}\left(z_{i k}-z_{j k}\right)}{\sum_{l=1}^{m} w_{l r}}\right)^{\alpha}, & \text { if } z_{i k} \geq z_{j k}, \\ -\lambda\left(\frac{\left(\sum_{l=1}^{m} w_{l r}\right)\left(z_{j k}-z_{i k}\right)}{w_{k r}}\right)^{\alpha}, & \text { if } z_{i k}<z_{j k} .\end{cases}
$$

Thus, when $\alpha=0.5$ and $\lambda=1 / \theta$ we obtain expression (1).

\section{Analysis of the TODIM method}

In this section we analyze the TODIM method from a double perspective. First, although this method is relatively easy to implement, we provide a simplified version of it. Second, we show two paradoxes affecting the weights of the model.

\footnotetext{
${ }^{3}$ Experimental studies carried out by Tversky and Kahneman [35] reveal median values of $\alpha=\beta=0.88$ and $\lambda=2.25$; see also Blavatskyy [4].
} 


\subsection{Simplification of the model}

The TODIM method is mainly based on the function $\Phi_{k}\left(A_{i}, A_{j}\right)$, which returns the dominance degree of alternative $A_{i}$ over alternative $A_{j}$ concerning criterion $C_{k}$. This function can be simplified taking into account that, for any $k \in M$,

$$
\frac{w_{k r}}{\sum_{l=1}^{m} w_{l r}}=\frac{w_{k} / w_{r}}{\sum_{l=1}^{m} w_{l} / w_{r}}=\frac{w_{k}}{\sum_{l=1}^{m} w_{l}}=w_{k},
$$

and, analogously,

$$
\frac{\sum_{l=1}^{m} w_{l r}}{w_{k r}}=\frac{1}{w_{k}} .
$$

Therefore, for each $i, j \in N$ and $k \in M, \Phi_{k}\left(A_{i}, A_{j}\right)$ can be written as

$$
\Phi_{k}\left(A_{i}, A_{j}\right)= \begin{cases}\sqrt{w_{k}\left(z_{i k}-z_{j k}\right)}, & \text { if } z_{i k} \geq z_{j k}, \\ -\frac{1}{\theta} \sqrt{\frac{z_{j k}-z_{i k}}{w_{k}},} & \text { if } z_{i k}<z_{j k} .\end{cases}
$$

In the same way, in the more general case, expression (3) becomes

$$
\Phi_{k}\left(A_{i}, A_{j}\right)= \begin{cases}\left(w_{k}\left(z_{i k}-z_{j k}\right)\right)^{\alpha}, & \text { if } z_{i k} \geq z_{j k}, \\ -\lambda\left(\frac{z_{j k}-z_{i k}}{w_{k}}\right)^{\alpha}, & \text { if } z_{i k}<z_{j k} .\end{cases}
$$

Notice that, for each $i \in N$, the values $\Phi_{k}\left(A_{i}, A_{j}\right)$ can be gathered in an $n \times m$ matrix of the form

$$
\boldsymbol{\Phi}\left(A_{i}\right)=\left(\begin{array}{llll}
\Phi_{1}\left(A_{i}, A_{1}\right) & \Phi_{2}\left(A_{i}, A_{1}\right) & \cdots & \Phi_{m}\left(A_{i}, A_{1}\right) \\
\Phi_{1}\left(A_{i}, A_{2}\right) & \Phi_{2}\left(A_{i}, A_{2}\right) & \cdots & \Phi_{m}\left(A_{i}, A_{2}\right) \\
\ldots \ldots \ldots \ldots \ldots \ldots \ldots \ldots \ldots \ldots \ldots \ldots \ldots \ldots & \ldots \ldots \ldots \ldots
\end{array}\right],
$$

where the $i$ th row is null. Moreover, the ranking of the alternatives can be carried out without the need to calculate the values $\xi\left(A_{i}\right)$; that is, by using the values $\Phi\left(A_{i}\right)$. Therefore, the TODIM method can be implemented through the following steps:

Step 1: For each $i, j \in N$ and $k \in M$, calculate

$$
\Phi_{k}\left(A_{i}, A_{j}\right)= \begin{cases}\sqrt{w_{k}\left(z_{i k}-z_{j k}\right)}, & \text { if } z_{i k} \geq z_{j k}, \\ -\frac{1}{\theta} \sqrt{\frac{z_{j k}-z_{i k}}{w_{k}},}, & \text { if } z_{i k}<z_{j k} .\end{cases}
$$


Step 2: For each $i \in N$, calculate the overall performance of alternative $A_{i}$,

$$
\Phi\left(A_{i}\right)=\sum_{j=1}^{n} \sum_{k=1}^{m} \Phi_{k}\left(A_{i}, A_{j}\right)
$$

which corresponds to the sum of all the elements of the matrix $\boldsymbol{\Phi}\left(A_{i}\right)$.

Step 3: Rank the alternatives according to the values $\Phi\left(A_{i}\right)$.

\subsection{Two paradoxes}

A paradox occurs when a multicriteria method produces an undesirable outcome, which may be regarded by some people as surprising or as counter-intuitive (see Felsenthal [12, p. 20]). We are going to show that the TODIM method is vulnerable to two paradoxes affecting the weights of the model. The first one has been pointed out by Lourenzutti and Krohling [27, p. 6462] (see also Lee and Shih [26]) by using the decision matrix of Table 2.

Table 2: First decision matrix (first paradox).

\begin{tabular}{lll}
\hline & $C_{1}$ & $C_{2}$ \\
\hline$A_{1}$ & 0 & 1 \\
$A_{2}$ & 1 & 0
\end{tabular}

Given a weight vector $\boldsymbol{w}=\left(w_{1}, w_{2}\right)$, it seems logical to think that $A_{2}>A_{1}$ if $w_{1}>w_{2}$, and $A_{1}>A_{2}$ when $w_{2}>w_{1}$. But, as we will see next, this does not always occur. When we apply the TODIM method by using the algorithm given in Subsection 3.1 we get $^{5}$

$$
\boldsymbol{\Phi}^{w}\left(A_{1}\right)=\left(\begin{array}{cc}
0 & 0 \\
-\frac{1}{\theta \sqrt{w_{1}}} & \sqrt{w_{2}}
\end{array}\right), \quad \boldsymbol{\Phi}^{w}\left(A_{2}\right)=\left(\begin{array}{cc}
\sqrt{w_{1}} & -\frac{1}{\theta \sqrt{w_{2}}} \\
0 & 0
\end{array}\right),
$$

\footnotetext{
${ }^{4}$ Notice that these authors explain the paradox through the expressions given in Section 2. However, for the sake of clarity, we will use the expressions given in Subsection 3.1.

${ }^{5}$ In the TODIM method, the expressions $\Phi_{k}\left(A_{i}, A_{j}\right), \boldsymbol{\Phi}\left(A_{i}\right)$, and $\Phi\left(A_{i}\right)$ depend on the decision problem $(\mathbf{Z}, \boldsymbol{w})$, although, for the sake of simplicity, we have avoided indicating this dependency in the notation. However, given that in some parts of the paper we are going to consider different weight vectors, we will use the name of the vector as a superscript to the letters or symbols when necessary to avoid misunderstandings.
} 
and the overall performances of these alternatives are

$$
\Phi^{w}\left(A_{1}\right)=\sqrt{w_{2}}-\frac{1}{\theta \sqrt{w_{1}}}, \quad \Phi^{w}\left(A_{2}\right)=\sqrt{w_{1}}-\frac{1}{\theta \sqrt{w_{2}}} .
$$

Since $w_{2}$ is in the denominator of the second addend of $\Phi^{w}\left(A_{2}\right)$, small values of $w_{2}$ involve large values of the expression $1 /\left(\theta \sqrt{w_{2}}\right)$, and, consequently, $\Phi^{w}\left(A_{2}\right)$ takes smaller values than $\Phi^{w}\left(A_{1}\right)$. For instance, when $\theta=1$ and $\boldsymbol{w}=(0.9,0.1)$, we obtain $\Phi^{w}\left(A_{1}\right)=-0.738>-2.213=\Phi^{w}\left(A_{2}\right)$. In fact, fixed $\theta$, it is possible to get the weight vectors $\boldsymbol{w}$ for which $A_{1}>^{w} A_{2}$. Assuming that $w_{1}>w_{2}$, we have

$$
\begin{aligned}
\Phi^{w}\left(A_{1}\right)>\Phi^{w}\left(A_{2}\right) & \Leftrightarrow \sqrt{w_{2}}-\frac{1}{\theta \sqrt{w_{1}}}>\sqrt{w_{1}}-\frac{1}{\theta \sqrt{w_{2}}} \Leftrightarrow \theta w_{2} \sqrt{w_{1}}-\sqrt{w_{2}}>\theta w_{1} \sqrt{w_{2}}-\sqrt{w_{1}} \\
& \Leftrightarrow \theta \sqrt{w_{1}} \sqrt{w_{2}}\left(\sqrt{w_{1}}-\sqrt{w_{2}}\right)<\left(\sqrt{w_{1}}-\sqrt{w_{2}}\right) \Leftrightarrow \theta<\frac{1}{\sqrt{w_{1} w_{2}}} .
\end{aligned}
$$

As we can see in the above expression, the paradox appears as $w_{2}$ approaches zero. This paradox can also be shown by using a more general decision matrix ${ }^{6}$. For instance, consider the decision matrix given in Table 3.

Table 3: Second decision matrix (first paradox).

\begin{tabular}{lllll}
\hline & $C_{1}$ & $C_{2}$ & $C_{3}$ & $C_{4}$ \\
\hline$A_{1}$ & 0.8 & 0 & 1 & 1 \\
$A_{2}$ & 0.7 & 0.4 & 0.9 & 0.2 \\
$A_{3}$ & 0.7 & 0.4 & 0.2 & 0.9 \\
$A_{4}$ & 0 & 1 & 0.3 & 0.3 \\
$A_{5}$ & 1 & 0.6 & 0 & 0 \\
\hline
\end{tabular}

When we compare alternatives $A_{2}$ and $A_{3}$ we see that both alternatives have the same scores with respect to criteria $C_{1}$ and $C_{2}$, while the scores of criteria $C_{3}$ and $C_{4}$ are switched. Moreover, in order to avoid that the order between $A_{2}$ and $A_{3}$ may be affected by other alternatives ${ }^{7}$, the scores of each one of the remaining alternatives with respect to criteria $C_{3}$ and $C_{4}$ will be held equal. Given the symmetry of the decision matrix with respect to alternatives $A_{2}$ and $A_{3}$ and criteria $C_{3}$ and $C_{4}$, it seems logical to think that

\footnotetext{
${ }^{6}$ We use this new decision matrix in order to illustrate the weight consistency property, which will be established in Section 4.

${ }^{7}$ This shortcoming is known as the rank reversal problem and it may be suffered by pairwise comparison methods; see, for instance, De Keyser and Peeters [9], Wang and Triantaphyllou [38], and Verly and De Smet [36].
} 
$\Phi\left(A_{2}\right) \geq \Phi\left(A_{3}\right)$ when $w_{3} \geq w_{4}$ while $\Phi\left(A_{3}\right) \geq \Phi\left(A_{2}\right)$ if $w_{4} \geq w_{3}$. However, if we take, for instance, $\theta=1$ and $\boldsymbol{w}=(0.2,0.2,0.5,0.1)$, we get $\Phi\left(A_{2}\right)=-8.977<-6.776=\Phi\left(A_{3}\right)$.

Let us now look at the second paradox. Consider a decision problem with three alternatives and two criteria, where the decision matrix is given in Table 4 and the weight vector is $\boldsymbol{w}=(0.7,0.3)$. When we

Table 4: Decision matrix (second paradox).

\begin{tabular}{lll}
\hline & $C_{1}$ & $C_{2}$ \\
\hline$A_{1}$ & 1 & 0.6 \\
$A_{2}$ & 0.4 & 1 \\
$A_{3}$ & 0 & 0 \\
\hline
\end{tabular}

apply the TODIM method with $\theta=1$ the matrices $\boldsymbol{\Phi}^{\boldsymbol{w}}\left(A_{1}\right)$ and $\boldsymbol{\Phi}^{\boldsymbol{w}}\left(A_{2}\right)$ corresponding to $A_{1}$ and $A_{2}$ are

$$
\boldsymbol{\Phi}^{w}\left(A_{1}\right)=\left(\begin{array}{cc}
0 & 0 \\
0.648 & -1.155 \\
0.837 & 0.424
\end{array}\right), \quad \boldsymbol{\Phi}^{w}\left(A_{2}\right)=\left(\begin{array}{cc}
-0.926 & 0.346 \\
0 & 0 \\
0.529 & 0.548
\end{array}\right),
$$

and the overall performances of these alternatives are $\Phi^{w}\left(A_{1}\right)=0.754$ and $\Phi^{w}\left(A_{2}\right)=0.497$; hence $A_{1}>^{w}$ $A_{2}$. Consider now the weight vector $\boldsymbol{w}^{\prime}=(0.9,0.1)$. Given that $A_{1}$ has a better performance value than $A_{2}$ with respect to criterion $C_{1}$ (and the weight of $C_{1}$ has increased) and $A_{1}$ has a worse performance value than $A_{2}$ with respect to criterion $C_{2}$ (and the weight of $C_{2}$ has decreased), it seems reasonable to think that the relationship between $A_{1}$ and $A_{2}$ should not change with this new weight vector; that is, $A_{1}>^{w^{\prime}} A_{2}$. However, when we calculate the dominance degrees among the alternatives, we get

$$
\boldsymbol{\Phi}^{w^{\prime}}\left(A_{1}\right)=\left(\begin{array}{cc}
0 & 0 \\
0.735 & -2 \\
0.949 & 0.245
\end{array}\right), \quad \boldsymbol{\Phi}^{w^{\prime}}\left(A_{2}\right)=\left(\begin{array}{cc}
-0.816 & 0.2 \\
0 & 0 \\
0.6 & 0.316
\end{array}\right),
$$

and the overall performances of these alternatives are $\Phi^{w^{\prime}}\left(A_{1}\right)=-0.071$ and $\Phi^{w^{\prime}}\left(A_{2}\right)=0.3$; so $A_{2}>^{w^{\prime}} A_{1}$. This change is mainly due to the difference between $\Phi_{2}^{w}\left(A_{1}, A_{2}\right)=-1.155$ and $\Phi_{2}^{w^{\prime}}\left(A_{1}, A_{2}\right)=-2$. Given that the new weight $w_{2}^{\prime}=0.1$ is less than the initial weight $w_{2}=0.3, \Phi_{2}^{w^{\prime}}\left(A_{1}, A_{2}\right)$ should also be less than $\Phi_{2}^{w}\left(A_{1}, A_{2}\right)$ in absolute value. However, it is considerably higher due to $\Phi_{k}\left(A_{i}, A_{j}\right)$ is, in the case of losses, inversely proportional to the weight $w_{k}$ (it appears in the denominator of the expression). 
The previous shortcoming may also appear in real MCDM problems. For instance, consider the case study carried out by Gomes and Rangel [17], where the TODIM method was applied to rank fifteen properties located in different neighborhoods in the city of Volta Redonda (Brazil) by using eight criteria: Location $\left(C_{1}\right)$, Constructed area $\left(C_{2}\right)$, Construction quality $\left(C_{3}\right)$, State of conservation $\left(C_{4}\right)$, Number of garage spaces $\left(C_{5}\right)$, Number of rooms $\left(C_{6}\right)$, Attractions $\left(C_{7}\right)$, and Security $\left(C_{8}\right)$. The decision matrix utilized by these authors $^{8}$ is shown in Table 5 and $\boldsymbol{w}=(0.25,0.15,0.10,0.20,0.05,0.10,0.05,0.10)$ was the weight vector used. The TODIM method was applied with $\theta=1$, and the two best properties were $A_{5}$ and $A_{14}\left(A_{5}>^{w} A_{14}\right.$, see

Table 5: Decision matrix taken from Gomes and Rangel [17].

\begin{tabular}{lllllllll}
\hline & $C_{1}$ & $C_{2}$ & $C_{3}$ & $C_{4}$ & $C_{5}$ & $C_{6}$ & $C_{7}$ & $C_{8}$ \\
\hline$A_{1}$ & 0.068 & 0.103 & 0.1 & 0.075 & 0.045 & 0.069 & 0.174 & 0 \\
$A_{2}$ & 0.091 & 0.064 & 0.067 & 0.05 & 0.045 & 0.046 & 0.087 & 0 \\
$A_{3}$ & 0.068 & 0.123 & 0.033 & 0.05 & 0.091 & 0.057 & 0.043 & 0 \\
$A_{4}$ & 0.068 & 0.044 & 0.067 & 0.075 & 0.091 & 0.057 & 0.174 & 0 \\
$A_{5}$ & 0.114 & 0.127 & 0.1 & 0.1 & 0.182 & 0.103 & 0.043 & 0.143 \\
$A_{6}$ & 0.045 & 0.031 & 0.067 & 0.075 & 0.045 & 0.057 & 0.043 & 0 \\
$A_{7}$ & 0.023 & 0.03 & 0.033 & 0.025 & 0.045 & 0.046 & 0 & 0.143 \\
$A_{8}$ & 0.114 & 0.028 & 0.067 & 0.075 & 0.045 & 0.069 & 0 & 0.143 \\
$A_{9}$ & 0.045 & 0.043 & 0.067 & 0.075 & 0 & 0.069 & 0 & 0 \\
$A_{10}$ & 0.045 & 0.042 & 0.033 & 0.075 & 0.045 & 0.057 & 0.043 & 0 \\
$A_{11}$ & 0.091 & 0.099 & 0.067 & 0.05 & 0.091 & 0.08 & 0.13 & 0.143 \\
$A_{12}$ & 0.023 & 0.032 & 0.033 & 0.025 & 0.045 & 0.057 & 0.087 & 0 \\
$A_{13}$ & 0.045 & 0.057 & 0.1 & 0.075 & 0.091 & 0.069 & 0.043 & 0.143 \\
$A_{14}$ & 0.068 & 0.113 & 0.1 & 0.075 & 0.091 & 0.092 & 0.087 & 0.143 \\
$A_{15}$ & 0.091 & 0.064 & 0.067 & 0.1 & 0.045 & 0.069 & 0.043 & 0.143 \\
\hline
\end{tabular}

Table 10 in Gomes and Rangel [17]). The overall performances of these alternatives (without normalizing) were $\Phi^{w}\left(A_{5}\right)=0.317$ and $\Phi^{w}\left(A_{14}\right)=-2.934$.

When we compare $A_{5}$ and $A_{14}$, we note that $A_{5}$ has a better performance value than $A_{14}$ with respect to

\footnotetext{
${ }^{8}$ The normalization was carried out through the expression $z_{i k}=\frac{x_{i k}}{\sum_{j=1}^{n} x_{j k}}$.
} 
criteria $C_{1}, C_{2}, C_{4}, C_{5}$ and $C_{6}$; they have the same performance value with respect to criteria $C_{3}$ and $C_{8}$, and only with respect to criterion $C_{7}$ did property $A_{5}$ have a worse performance value than $A_{14}$. Hence, one would expect that a decrease in the weight of criterion $C_{7}$ and an increase in the weight of one of criteria $C_{1}, C_{2}, C_{4}, C_{5}$ or $C_{6}$ should preserve the order between $A_{5}$ and $A_{14}$.

However, this is not the case, and as $w_{7}$ approaches zero, the order between $A_{5}$ and $A_{14}$ is reversed. For instance, in Table 6 we show the overall performances of $A_{5}$ and $A_{14}$ when the weight of criterion $C_{7}$ decreases by 0.04 units and the weight of one of criteria $C_{1}, C_{2}, C_{4}, C_{5}$ or $C_{6}$ increases by the same amount ${ }^{9}$. Notice that, in all cases, $A_{14}>^{w^{\prime}} A_{5}$.

Table 6: Overall performances by using different weight vectors.

\begin{tabular}{llllll}
\hline & $C_{1}$ & $C_{2}$ & $C_{4}$ & $C_{5}$ & $C_{6}$ \\
\hline$\Phi^{w^{\prime}}\left(A_{5}\right)$ & -8.756 & -8.706 & -8.765 & -8.497 & -8.712 \\
$\Phi^{w^{\prime}}\left(A_{14}\right)$ & -7.440 & -7.405 & -7.505 & -7.109 & -7.426 \\
\hline
\end{tabular}

In fact, it is possible, by using mathematical software, to obtain the values of the weight of criterion $C_{7}$ for which the order between $A_{5}$ and $A_{14}$ is reversed. These values, given in terms of intervals, are shown in Table $7 .{ }^{10}$

Table 7: Values of $w_{7}^{\prime}$ for which $A_{14}>^{w^{\prime}} A_{5}$.

\begin{tabular}{llllll}
\hline & $C_{1}$ & $C_{2}$ & $C_{4}$ & $C_{5}$ & $C_{6}$ \\
\hline$w_{7}^{\prime}$ & $(0,0.01408)$ & $(0,0.01403)$ & $(0,0.01388)$ & $(0,0.01438)$ & $(0,0.01398)$ \\
\hline
\end{tabular}

As we will see in the next section, the previous paradoxes can be avoided by using nondecreasing functions in the definition of $\Phi_{k}\left(A_{i}, A_{j}\right)$.

\footnotetext{
${ }^{9}$ For instance, the overall performances of $A_{5}$ and $A_{14}$ that appear under the label $C_{6}$ are obtained by using the weight vector $\boldsymbol{w}^{\prime}=(0.25,0.15,0.10,0.20,0.05,0.14,0.01,0.10)$.

${ }^{10}$ Numbers have been rounded to five decimal places. The interval $(0,0.01408)$ that appears under the label $C_{1}$ means that if $w_{7}^{\prime} \in(0,0.01408), w_{1}^{\prime}=w_{1}+\left(w_{7}-w_{7}^{\prime}\right)=0.3-w_{7}^{\prime}$, and $w_{i}^{\prime}=w_{i}$ for any $i \neq 1,7$, then $A_{14}>^{w^{\prime}} A_{5}$.
} 


\section{The generalized TODIM method and its properties}

The paradoxes shown in the previous section bring into question the importance of the weights. Hence, it seems necessary to introduce certain properties which prevent the occurrence of such paradoxes. These properties, which we will call weight consistency and weight monotonicity, will be defined for any multicriteria method.

Definition 1. Let $F$ be a multicriteria method. $F$ is weight consistent if for each decision problem $(\mathbf{Z}, \boldsymbol{w})$ where there exist two alternatives $A_{p}$ and $A_{q}$ and two criteria $C_{r}$ and $C_{s}$ such that $z_{p r}=z_{q s} \geq z_{p s}=z_{q r}$, $z_{p k}=z_{q k}$ for all $k \neq r, s$ and $z_{j r}=z_{j s}$ for all $j \neq p, q$, the following condition is satisfied:

$$
w_{r} \geq w_{s} \Rightarrow A_{p} \succcurlyeq A_{q} .
$$

Definition 2. Let $F$ be a multicriteria method. $F$ satisfies weight monotonicity if for each decision problem $(\mathbf{Z}, \boldsymbol{w})$, for each pair of alternatives $A_{p}$ and $A_{q}$ and each pair of criteria $C_{r}$ and $C_{s}$ such that $z_{p r} \geq z_{q r}$ and $z_{p s} \leq z_{q s}$, and for each normalized weight vector $\boldsymbol{w}^{\prime}$ such that $w_{k}^{\prime}=w_{k}(k \neq r, s), w_{r}^{\prime}=w_{r}+\varepsilon$, and $w_{s}^{\prime}=w_{s}-\varepsilon$, where $0<\varepsilon<w_{s}$, the following condition is satisfied:

$$
A_{p} \succcurlyeq^{w} A_{q} \Rightarrow A_{p} \succcurlyeq^{w^{\prime}} A_{q}
$$

As we have previously seen, the TODIM method does not satisfy the above properties when the function $\Phi_{k}\left(A_{i}, A_{j}\right)$ is defined through expression (4). Therefore, we are going to introduce a more general form of the function $\Phi_{k}\left(A_{i}, A_{j}\right)$ :

$$
\Phi_{k}\left(A_{i}, A_{j}\right)=\left\{\begin{array}{cc}
g_{1}\left(w_{k}\right) f_{1}\left(z_{i k}-z_{j k}\right), & \text { if } z_{i k} \geq z_{j k}, \\
-g_{2}\left(w_{k}\right) f_{2}\left(z_{j k}-z_{i k}\right), & \text { if } z_{i k}<z_{j k},
\end{array}\right.
$$

where $g_{1}, g_{2}:(0,1) \longrightarrow(0,+\infty), f_{1}, f_{2}:[0,1] \longrightarrow[0,+\infty)$ and $f_{1}(0)=f_{2}(0)=0$. Notice that expression (4) is obtained when $g_{1}(x)=f_{1}(x)=f_{2}(x)=\sqrt{x}$ and $g_{2}(x)=1 /(\theta \sqrt{x})$. Likewise, expression (5) can be recovered if $g_{1}(x)=f_{1}(x)=f_{2}(x)=x^{\alpha}$ and $g_{2}(x)=\lambda / x^{\alpha}$.

Hence, the implementation of the generalized TODIM method entails the following steps:

Step 1: For each $i, j \in N$ and $k \in M$, calculate

$$
\Phi_{k}\left(A_{i}, A_{j}\right)=\left\{\begin{array}{cc}
g_{1}\left(w_{k}\right) f_{1}\left(z_{i k}-z_{j k}\right), & \text { if } z_{i k} \geq z_{j k}, \\
-g_{2}\left(w_{k}\right) f_{2}\left(z_{j k}-z_{i k}\right), & \text { if } z_{i k}<z_{j k},
\end{array}\right.
$$

where $g_{1}, g_{2}, f_{1}$ and $f_{2}$ satisfy the properties listed above. 
Step 2: For each $i \in N$, calculate the overall performance of alternative $A_{i}$,

$$
\Phi\left(A_{i}\right)=\sum_{j=1}^{n} \sum_{k=1}^{m} \Phi_{k}\left(A_{i}, A_{j}\right),
$$

which corresponds to the sum of all the elements of the matrix $\boldsymbol{\Phi}\left(A_{i}\right)$.

Step 3: Rank the alternatives according to the values $\Phi\left(A_{i}\right)$.

As we will see in Theorems 1 and 2, we can guarantee the weight consistency and the weight monotonicity of the generalized TODIM method when the functions $g_{1}, g_{2}, f_{1}$ and $f_{2}$ are nondecreasing ${ }^{11}$. The following technical lemma will be useful in the proof of both theorems.

Lemma 1. Consider the generalized TODIM method defined through expression (7) and let $(\mathbf{Z}, \boldsymbol{w})$ be a decision problem where there exist two alternatives $A_{p}$ and $A_{q}$ and two criteria $C_{r}$ and $C_{s}$ such that $z_{p r} \geq z_{q r}$, $z_{p s} \leq z_{q s}$. If $f_{1}$ and $f_{2}$ are nondecreasing functions, then

$$
\Phi\left(A_{p}\right)-\Phi\left(A_{q}\right)=K+g_{1}\left(w_{r}\right) K_{1}+g_{2}\left(w_{r}\right) K_{2}-g_{1}\left(w_{s}\right) K_{3}-g_{2}\left(w_{s}\right) K_{4},
$$

where

$$
\begin{aligned}
K & =\sum_{k \neq r, s} \sum_{j=1}^{n} \Phi_{k}\left(A_{p}, A_{j}\right)-\sum_{k \neq r, s} \sum_{j=1}^{n} \Phi_{k}\left(A_{q}, A_{j}\right), \\
K_{1} & =\sum_{j \in J_{p r}^{+}} f_{1}\left(z_{p r}-z_{j r}\right)-\sum_{j \in J_{q r}^{+}} f_{1}\left(z_{q r}-z_{j r}\right), \\
K_{2} & =\sum_{j \in J_{q r}^{-}} f_{2}\left(z_{j r}-z_{q r}\right)-\sum_{j \in J_{p r}^{-}} f_{2}\left(z_{j r}-z_{p r}\right), \\
K_{3}= & \sum_{j \in J_{q s}^{+}} f_{1}\left(z_{q s}-z_{j s}\right)-\sum_{j \in J_{p s}^{+}} f_{1}\left(z_{p s}-z_{j s}\right), \\
K_{4}= & \sum_{j \in J_{p s}^{-}} f_{2}\left(z_{j s}-z_{p s}\right)-\sum_{j \in J_{q s}^{-}} f_{2}\left(z_{j s}-z_{q s}\right),
\end{aligned}
$$

$K_{1}, K_{2}, K_{3}, K_{4} \geq 0$ and, for each $i \in N$ and $k \in M$, the sets $J_{i k}^{+}$and $J_{i k}^{-}$are defined by

$$
J_{i k}^{+}=\left\{j \in N \mid z_{i k} \geq z_{j k}\right\}, \quad J_{i k}^{-}=\left\{j \in N \mid z_{i k}<z_{j k}\right\} .
$$

Proof. Let $(\mathbf{Z}, \boldsymbol{w})$ be a decision problem where there exist two alternatives $A_{p}$ and $A_{q}$ and two criteria $C_{r}$ and $C_{s}$ such that $z_{p r} \geq z_{q r}$ and $z_{p s} \leq z_{q s}$. Given $i \in N$ and $k \in M$, we define the following sets:

$$
J_{i k}^{+}=\left\{j \in N \mid z_{i k} \geq z_{j k}\right\}, \quad J_{i k}^{-}=\left\{j \in N \mid z_{i k}<z_{j k}\right\} .
$$

\footnotetext{
${ }^{11}$ It is worth noting that neither $g_{2}(x)=1 /(\theta \sqrt{x})$ nor $g_{2}(x)=\lambda / x^{\alpha}$ are nondecreasing functions.
} 
Notice that $J_{i k}^{+} \cup J_{i k}^{-}=N$ and $J_{i k}^{+} \cap J_{i k}^{-}=\varnothing$. By using these sets, the overall performance of alternative $A_{i}$ can be calculated by means of the following expression:

$$
\begin{aligned}
\Phi\left(A_{i}\right)= & \sum_{k \neq r, s} \sum_{j=1}^{n} \Phi_{k}\left(A_{i}, A_{j}\right)+\sum_{j=1}^{n} \Phi_{r}\left(A_{i}, A_{j}\right)+\sum_{j=1}^{n} \Phi_{s}\left(A_{i}, A_{j}\right) \\
= & \sum_{k \neq r, s} \sum_{j=1}^{n} \Phi_{k}\left(A_{i}, A_{j}\right)+g_{1}\left(w_{r}\right) \sum_{j \in J_{i r}^{+}} f_{1}\left(z_{i r}-z_{j r}\right)-g_{2}\left(w_{r}\right) \sum_{j \in J_{i r}^{-}} f_{2}\left(z_{j r}-z_{i r}\right) \\
& +g_{1}\left(w_{s}\right) \sum_{j \in J_{i s}^{+}} f_{1}\left(z_{i s}-z_{j s}\right)-g_{2}\left(w_{s}\right) \sum_{j \in J_{i s}^{-}} f_{2}\left(z_{j s}-z_{i s}\right) .
\end{aligned}
$$

Consider now $A_{p}$ and $A_{q}$. By the previous identity we have

$$
\begin{aligned}
\Phi\left(A_{p}\right) & -\Phi\left(A_{q}\right)=\sum_{k \neq r, s} \sum_{j=1}^{n} \Phi_{k}\left(A_{p}, A_{j}\right)-\sum_{k \neq r, s} \sum_{j=1}^{n} \Phi_{k}\left(A_{q}, A_{j}\right) \\
& +g_{1}\left(w_{r}\right)\left(\sum_{j \in J_{p r}^{+}} f_{1}\left(z_{p r}-z_{j r}\right)-\sum_{j \in J_{q r}^{+}} f_{1}\left(z_{q r}-z_{j r}\right)\right)+g_{2}\left(w_{r}\right)\left(\sum_{j \in J_{q r}^{-}} f_{2}\left(z_{j r}-z_{q r}\right)-\sum_{j \in J_{p r}^{-}} f_{2}\left(z_{j r}-z_{p r}\right)\right) \\
& -g_{1}\left(w_{s}\right)\left(\sum_{j \in J_{q s}^{+}} f_{1}\left(z_{q s}-z_{j s}\right)-\sum_{j \in J_{p s}^{+}} f_{1}\left(z_{p s}-z_{j s}\right)\right)-g_{2}\left(w_{s}\right)\left(\sum_{j \in J_{p s}^{-}} f_{2}\left(z_{j s}-z_{p s}\right)-\sum_{j \in J_{q s}^{-}} f_{2}\left(z_{j s}-z_{q s}\right)\right),
\end{aligned}
$$

that is,

$$
\Phi\left(A_{p}\right)-\Phi\left(A_{q}\right)=K+g_{1}\left(w_{r}\right) K_{1}+g_{2}\left(w_{r}\right) K_{2}-g_{1}\left(w_{s}\right) K_{3}-g_{2}\left(w_{s}\right) K_{4} .
$$

Now, we are going to prove that $K_{1} \geq 0$. Since $z_{p r} \geq z_{q r}$ and $z_{p s} \leq z_{q s}$, it is straightforward to check that we have the following relationships:

$$
J_{q r}^{+} \subseteq J_{p r}^{+}, \quad J_{p r}^{-} \subseteq J_{q r}^{-}, \quad J_{p s}^{+} \subseteq J_{q s}^{+}, \quad J_{q s}^{-} \subseteq J_{p s}^{-},
$$

and, therefore,

$$
K_{1}=\sum_{j \in J_{p r}^{+}} f_{1}\left(z_{p r}-z_{j r}\right)-\sum_{j \in J_{q r}^{+}} f_{1}\left(z_{q r}-z_{j r}\right)=\sum_{j \in J_{q r}^{+}}\left(f_{1}\left(z_{p r}-z_{j r}\right)-f_{1}\left(z_{q r}-z_{j r}\right)\right)+\sum_{j \in J_{p r}^{+} J_{q r}^{+}} f_{1}\left(z_{p r}-z_{j r}\right) \geq 0,
$$

where the last inequality is obtained by taking into account that $z_{p r}-z_{j r} \geq z_{q r}-z_{j r}$ and $f_{1}$ is nondecreasing. In the same way, the following inequalities can be proven:

$$
\begin{aligned}
& K_{2}=\sum_{j \in J_{q r}^{-}} f_{2}\left(z_{j r}-z_{q r}\right)-\sum_{j \in J_{p r}^{-}} f_{2}\left(z_{j r}-z_{p r}\right) \geq 0, \\
& K_{3}=\sum_{j \in J_{q s}^{+}} f_{1}\left(z_{q s}-z_{j s}\right)-\sum_{j \in J_{p s}^{+}} f_{1}\left(z_{p s}-z_{j s}\right) \geq 0, \\
& K_{4}=\sum_{j \in J_{p s}^{-}} f_{2}\left(z_{j s}-z_{p s}\right)-\sum_{j \in J_{q s}^{-}} f_{2}\left(z_{j s}-z_{q s}\right) \geq 0 .
\end{aligned}
$$


Theorem 1. Consider the generalized TODIM method defined through expression (7). If $f_{1}, f_{2}, g_{1}$ and $g_{2}$ are nondecreasing functions, then the TODIM method is weight consistent.

Proof. Let $(\mathbf{Z}, \boldsymbol{w})$ be a decision problem where there exist two alternatives $A_{p}$ and $A_{q}$ and two criteria $C_{r}$ and $C_{s}$ such that $z_{p r}=z_{q s} \geq z_{p s}=z_{q r}, z_{p k}=z_{q k}$ for all $k \neq r, s$, and $z_{j r}=z_{j s}$ for all $j \neq p, q$. Since $f_{1}$ and $f_{2}$ are nondecreasing, by Lemma 1 we have

$$
\Phi\left(A_{p}\right)-\Phi\left(A_{q}\right)=K+g_{1}\left(w_{r}\right) K_{1}+g_{2}\left(w_{r}\right) K_{2}-g_{1}\left(w_{s}\right) K_{3}-g_{2}\left(w_{s}\right) K_{4},
$$

where $K_{1}, K_{2}, K_{3}, K_{4} \geq 0$. Moreover, due to the conditions satisfied by $\mathbf{Z}$, it is easy to check that $K=0$, $K_{1}=K_{3}$ and $K_{2}=K_{4}$. Hence,

$$
\Phi\left(A_{p}\right)-\Phi\left(A_{q}\right)=\left(g_{1}\left(w_{r}\right)-g_{1}\left(w_{s}\right)\right) K_{1}+\left(g_{2}\left(w_{r}\right)-g_{2}\left(w_{s}\right)\right) K_{2} .
$$

Finally, since $g_{1}$ and $g_{2}$ are nondecreasing, when $w_{r} \geq w_{s}$ we get $\Phi\left(A_{p}\right)-\Phi\left(A_{q}\right) \geq 0$, i.e., $A_{p} \succcurlyeq A_{q}$.

Theorem 2. Consider the generalized TODIM method defined through expression (7). If $f_{1}, f_{2}, g_{1}$ and $g_{2}$ are nondecreasing functions, then the TODIM method satisfies weight monotonicity.

Proof. Let $(\mathbf{Z}, \boldsymbol{w})$ be a decision problem where there exist two alternatives $A_{p}$ and $A_{q}$ and two criteria $C_{r}$ and $C_{s}$ such that $z_{p r} \geq z_{q r}, z_{p s} \leq z_{q s}$ and $A_{p} \succcurlyeq^{w} A_{q}$, i.e., $\Phi^{w}\left(A_{p}\right) \geq \Phi^{w}\left(A_{q}\right)$. Since $f_{1}$ and $f_{2}$ are nondecreasing, by Lemma 1 we have ${ }^{12}$

$$
\Phi^{w}\left(A_{p}\right)-\Phi^{w}\left(A_{q}\right)=K^{w}+g_{1}\left(w_{r}\right) K_{1}+g_{2}\left(w_{r}\right) K_{2}-g_{1}\left(w_{s}\right) K_{3}-g_{2}\left(w_{s}\right) K_{4} \geq 0,
$$

that is,

$$
K^{w}+g_{1}\left(w_{r}\right) K_{1}+g_{2}\left(w_{r}\right) K_{2} \geq g_{1}\left(w_{s}\right) K_{3}+g_{2}\left(w_{s}\right) K_{4},
$$

where $K_{1}, K_{2}, K_{3}, K_{4} \geq 0$. Consider now $0<\varepsilon<w_{s}$ and a weight vector $\boldsymbol{w}^{\prime}$ defined by $w_{k}^{\prime}=w_{k}(k \neq r, s)$, $w_{r}^{\prime}=w_{r}+\varepsilon$, and $w_{s}^{\prime}=w_{s}-\varepsilon$. Since $g_{1}$ and $g_{2}$ are nondecreasing, by replacing $w_{r}$ by $w_{r}+\varepsilon$ and $w_{s}$ by $w_{s}-\varepsilon$, we also get

$$
K^{w^{\prime}}+g_{1}\left(w_{r}^{\prime}\right) K_{1}+g_{2}\left(w_{r}^{\prime}\right) K_{2}>g_{1}\left(w_{s}^{\prime}\right) K_{3}+g_{2}\left(w_{s}^{\prime}\right) K_{4},
$$

that is,

$$
\Phi^{w^{\prime}}\left(A_{p}\right)-\Phi^{w^{\prime}}\left(A_{q}\right)=K^{w^{\prime}}+g_{1}\left(w_{r}^{\prime}\right) K_{1}+g_{2}\left(w_{r}^{\prime}\right) K_{2}-g_{1}\left(w_{s}^{\prime}\right) K_{3}-g_{2}\left(w_{s}^{\prime}\right) K_{4} \geq 0,
$$

where the first equality is obtained from Lemma 1 . Hence, $A_{p} \succcurlyeq{ }^{w^{\prime}} A_{q}$.

\footnotetext{
${ }^{12}$ Notice that $K_{1}, K_{2}, K_{3}, K_{4}$ do not depend on the weights $w_{k}, k \in M$, and $K$ only depends on the weights $w_{k}$, where $k \neq r, s$.
} 


\section{Specific cases of the generalized TODIM method}

The functions $g_{1}, g_{2}, f_{1}$ and $f_{2}$ that appear in expression (7) offer a lot of possibilities in the implementation of the generalized TODIM method. For instance, some particular cases of the generalized TODIM method have already been used in the literature. Thus, Lourenzutti and Krohling [27, p. 6462] consider

$$
\Phi_{k}\left(A_{i}, A_{j}\right)= \begin{cases}\sqrt{w_{k}\left(z_{i k}-z_{j k}\right)}, & \text { if } z_{i k} \geq z_{j k}, \\ -\frac{1}{\theta} \sqrt{w_{k}\left(z_{j k}-z_{i k}\right)}, & \text { if } z_{i k}<z_{j k},\end{cases}
$$

which is obtained from expression (7) when $g_{1}(x)=f_{1}(x)=\sqrt{x}, g_{2}(x)=(1 / \theta) \sqrt{x}$ and $f_{2}(x)=\sqrt{x}$ (or, alternatively, $g_{2}(x)=\sqrt{x}$ and $\left.f_{2}(x)=(1 / \theta) \sqrt{x}\right)$, while if we take $g_{1}(x)=f_{1}(x)=x^{\alpha}, g_{2}(x)=\lambda x^{\beta}$ and $f_{2}(x)=x^{\beta}$ (or, alternatively, $g_{2}(x)=x^{\beta}$ and $f_{2}(x)=\lambda x^{\beta}$ ), with $\alpha=\beta=0.88$ and $\lambda=2.25$, we obtain the expression used by Lee and Shih [26, p. 909],

$$
\Phi_{k}\left(A_{i}, A_{j}\right)= \begin{cases}\left(w_{k}\left(z_{i k}-z_{j k}\right)\right)^{\alpha}, & \text { if } z_{i k} \geq z_{j k}, \\ -\lambda\left(w_{k}\left(z_{j k}-z_{i k}\right)\right)^{\beta}, & \text { if } z_{i k}<z_{j k} .\end{cases}
$$

Notice that the generalized TODIM method allows us to obtain the same ordering of the alternatives as the SAW method (see, for instance, Hwang and Yoon [21]). Thus, when $g_{1}(x)=g_{2}(x)=f_{1}(x)=f_{2}(x)=x$, we have $\Phi_{k}\left(A_{i}, A_{j}\right)=w_{k}\left(z_{i k}-z_{j k}\right)$ for any $i, j \in N$ and $k \in M$. Therefore, for each $i \in N$,

$$
\Phi\left(A_{i}\right)=\sum_{k=1}^{m} \sum_{j=1}^{n} \Phi_{k}\left(A_{i}, A_{j}\right)=\sum_{k=1}^{m} w_{k}\left(n z_{i k}-\sum_{j=1}^{n} z_{j k}\right)=n \sum_{k=1}^{m} w_{k}\left(z_{i k}-\bar{z}_{\bullet k}\right),
$$

where $\bar{z}_{\bullet k}$ is the mean of the elements of the $k$ th column of the matrix $\mathbf{Z}$. Hence,

$$
\Phi\left(A_{p}\right) \geq \Phi\left(A_{q}\right) \Leftrightarrow \sum_{k=1}^{m} w_{k}\left(z_{p k}-\bar{z}_{\bullet k}\right) \geq \sum_{k=1}^{m} w_{k}\left(z_{q k}-\bar{z}_{\bullet k}\right) \Leftrightarrow \sum_{k=1}^{m} w_{k} z_{p k} \geq \sum_{k=1}^{m} w_{k} z_{q k} .
$$

It is also worth noting that, under certain hypotheses, the PROMETHEE II method is also a specific case of the generalized TODIM method. The PROMETHEE II method, which was developed by Brans [5] (see also Brans and Vincke [7] and Brans and De Smet [6]), provides a complete ranking among the alternatives (in contrast to PROMETHEE I, which only returns a partial ranking). When it comes to describing the PROMETHEE II method, we can assume, without loss of generality, that all criteria are to be maximized ${ }^{13}$. Using our notation, the PROMETHEE II method can be implemented by means of the following steps (see, for instance, Behzadian et al. [1]):

\footnotetext{
${ }^{13}$ If criterion $C_{k}$ is to be minimized, it is to be maximized when we use the values $-x_{i k}$ for all $i \in N$. Notice that this procedure is equivalent to consider the preference function defined by expression (6.9) in Brans and De Smet [6, p. 194].
} 
Step 1: For each $i, j \in N$ and $k \in M$, calculate the degree to which $A_{i}$ is preferred to $A_{j}$ with respect to criterion $C_{k}$; that is,

$$
P_{k}\left(A_{i}, A_{j}\right)=F_{k}\left(x_{i k}-x_{j k}\right)
$$

where $F_{k}: \mathbb{R} \longrightarrow[0,1]$ is a nondecreasing function such that $F_{k}(x)=0$ when $x \leq 0$. Some examples of functions satisfying the previous properties are shown in Brans and De Smet [6, p. 195].

Step 2: For each $i, j \in N$ calculate the degree to which $A_{i}$ is preferred to $A_{j}$ over all criteria; i.e.,

$$
\pi\left(A_{i}, A_{j}\right)=\sum_{k=1}^{m} w_{k} P_{k}\left(A_{i}, A_{j}\right)
$$

Step 3: For each $i \in N$ calculate the net outranking flow; that is,

$$
\phi\left(A_{i}\right)=\frac{1}{n-1} \sum_{j=1}^{n}\left(\pi\left(A_{i}, A_{j}\right)-\pi\left(A_{j}, A_{i}\right)\right) .
$$

Step 4: Rank the alternatives according to the values $\phi\left(A_{i}\right)$.

Notice that

$$
(n-1) \phi\left(A_{i}\right)=\sum_{j=1}^{n} \sum_{k=1}^{m} w_{k}\left(F_{k}\left(x_{i k}-x_{j k}\right)-F_{k}\left(x_{j k}-x_{i k}\right)\right),
$$

and, due to the properties satisfied by $F_{k}$, we have

$$
w_{k}\left(F_{k}\left(x_{i k}-x_{j k}\right)-F_{k}\left(x_{j k}-x_{i k}\right)\right)= \begin{cases}w_{k} F_{k}\left(x_{i k}-x_{j k}\right), & \text { if } x_{i k} \geq x_{j k}, \\ -w_{k} F_{k}\left(x_{j k}-x_{i k}\right), & \text { if } x_{i k}<x_{j k} .\end{cases}
$$

Since the values $\phi\left(A_{i}\right)$ and $(n-1) \phi\left(A_{i}\right)$ provide the same rank of alternatives, the PROMETHEE II method can be implemented by means of the following steps:

Step 1: For each $i, j \in N$ and $k \in M$, calculate

$$
\delta_{k}\left(A_{i}, A_{j}\right)= \begin{cases}w_{k} F_{k}\left(x_{i k}-x_{j k}\right), & \text { if } x_{i k} \geq x_{j k}, \\ -w_{k} F_{k}\left(x_{j k}-x_{i k}\right), & \text { if } x_{i k}<x_{j k} .\end{cases}
$$

Step 2: For each $i \in N$, calculate the overall performance of alternative $A_{i}$,

$$
\delta\left(A_{i}\right)=\sum_{j=1}^{n} \sum_{k=1}^{m} \delta_{k}\left(A_{i}, A_{j}\right) .
$$


Step 3: Rank the alternatives according to the values $\delta\left(A_{i}\right)$.

Therefore, when the decision matrix $\mathbf{X}$ is normalized and all functions $F_{k}$ are the same $\left(F_{k}=F\right.$ for all $k \in M$ ), the PROMETHEE II method is also a specific case of the generalized TODIM method; that is, $\delta_{k}\left(A_{i}, A_{j}\right)$ is obtained from expression (7) by taking $g_{1}(x)=g_{2}(x)=x$ and $f_{1}=f_{2}=\left.F\right|_{[0,1]}$.

Notice also that in PROMETHEE II we can get a very simple expression of $\delta\left(A_{i}\right)$ when all functions $F_{k}$ are type 1 functions ${ }^{14}$ (see Brans and De Smet [6, p. 195]); that is,

$$
F(x)= \begin{cases}0, & \text { if } x \leq 0 \\ 1, & \text { if } x>0\end{cases}
$$

Then the value $\delta_{k}\left(A_{i}, A_{j}\right)$ becomes

$$
\delta_{k}\left(A_{i}, A_{j}\right)= \begin{cases}w_{k}, & \text { if } x_{i k}>x_{j k}, \\ 0, & \text { if } x_{i k}=x_{j k}, \\ -w_{k}, & \text { if } x_{i k}<x_{j k},\end{cases}
$$

and, consequently, the overall performance of alternative $A_{i}$ is ${ }^{15}$

$$
\delta\left(A_{i}\right)=\sum_{k=1}^{m} \sum_{j=1}^{n} \delta_{k}\left(A_{i}, A_{j}\right)=\sum_{k=1}^{m} w_{k}\left(n_{i k}^{+}-n_{i k}^{-}\right),
$$

where $n_{i k}^{+}$and $n_{i k}^{-}$denote, respectively, the number of alternatives having a worse (better) score than $A_{i}$ with respect to criterion $C_{k}$ (roughly speaking, the number of victories and defeats of $A_{i}$ with respect to criterion $\left.C_{k}\right)$; i.e.,

$$
n_{i k}^{+}=\#\left\{j \in N \mid x_{i k}>x_{j k}\right\}, \quad n_{i k}^{-}=\#\left\{j \in N \mid x_{i k}<x_{j k}\right\},
$$

where the symbol \# denotes the cardinality of a set.

Next we show other interesting particular cases that we can get from expression (6). Since the weight vector $\boldsymbol{w}$ is previously fixed and reflects the importance of the criteria, it would seem wise not to modify

\footnotetext{
${ }^{14}$ The main advantage of this function is that the DM does not need to provide any parameter. See, for instance, Govindan et al. [19] for an example of a recent application of this function.

${ }^{15}$ Notice that $\delta\left(A_{i}\right)$ can be seen as the natural generalization of the score given by the adjusted Borda method (Black [2, pp. 7577], Black [3], Coughlin [8]), when the weights of the criteria are taken into account; that is, if $w_{k}=1 / m$ for all $k$, and for each criterion we ranking the alternatives according to their values, then we obtain the score given by the adjusted Borda method.
} 
these weights; that is, to consider $g_{1}(x)=g_{2}(x)=x$. In this case, the function $\Phi_{k}\left(A_{i}, A_{j}\right)$ becomes

$$
\Phi_{k}\left(A_{i}, A_{j}\right)=\left\{\begin{aligned}
w_{k} f_{1}\left(z_{i k}-z_{j k}\right), & \text { if } z_{i k} \geq z_{j k}, \\
-w_{k} f_{2}\left(z_{j k}-z_{i k}\right), & \text { if } z_{i k}<z_{j k} .
\end{aligned}\right.
$$

Now, according to the spirit of the subjective value function introduced by Tversky and Kahneman [35] (see expression (2)), we can distinguish the following cases: ${ }^{16}$

1. When $f_{1}(x)=x^{\alpha}$ and $f_{2}(x)=0$ we have

$$
\Phi_{k}\left(A_{i}, A_{j}\right)= \begin{cases}w_{k}\left(z_{i k}-z_{j k}\right)^{\alpha}, & \text { if } z_{i k} \geq z_{j k}, \\ 0, & \text { if } z_{i k}<z_{j k},\end{cases}
$$

that is, only gains are taken into account.

2. When $f_{1}(x)=0$ and $f_{2}(x)=\lambda x^{\beta}$ we get

$$
\Phi_{k}\left(A_{i}, A_{j}\right)= \begin{cases}0, & \text { if } z_{i k} \geq z_{j k}, \\ -\lambda w_{k}\left(z_{j k}-z_{i k}\right)^{\beta}, & \text { if } z_{i k}<z_{j k},\end{cases}
$$

i.e., only losses are considered. Note that, in this case, the value of $\lambda$ has no influence on the final ranking of the alternatives. So, we can take $\lambda=1$.

3. When $f_{1}(x)=x^{\alpha}$ and $f_{2}(x)=\lambda x^{\beta}$ we have

$$
\Phi_{k}\left(A_{i}, A_{j}\right)=\left\{\begin{aligned}
w_{k}\left(z_{i k}-z_{j k}\right)^{\alpha}, & \text { if } z_{i k} \geq z_{j k}, \\
-\lambda w_{k}\left(z_{j k}-z_{i k}\right)^{\beta}, & \text { if } z_{i k}<z_{j k} .
\end{aligned}\right.
$$

In the following we are going to apply some of the previous methods to the case study carried out by Gomes and Rangel [17]. In Table 8 we collect the ordering of the alternatives when the following methods are employed: the original TODIM method utilized by Gomes and Rangel [17] (Method 1); the generalized TODIM method with $\alpha=\beta=0.5$ and $\lambda=1$ by using expression (8) (Method 2), and by using expression (9) (Method 3); the generalized TODIM method with $\alpha=\beta=0.88$ and $\lambda=2.25$ by using expression (8) (Method 4), and by using expression (9) (Method 5); and the SAW method (Method 6).

Although the ordering of the alternatives is very similar in the six methods, it can be appreciated that Method 1 returns a slightly different ranking, especially in the position obtained by alternatives $A_{13}$ and $A_{15}$. This different behavior of the original TODIM method could be due to the fact that, in the case of losses, the value $\Phi_{k}\left(A_{i}, A_{j}\right)$ is inversely proportional to the weight $w_{k}$.

\footnotetext{
${ }^{16}$ In all cases, $0<\alpha, \beta<1$ and $\lambda>0$. Remember that if $\lambda>1$ the losses are amplified while if $\lambda<1$ the losses are attenuated.
} 
Table 8: Ranking of the alternatives for different multicriteria methods.

\begin{tabular}{lllllll}
\hline & Method 1 & Method 2 & Method 3 & Method 4 & Method 5 & Method 6 \\
\hline$A_{1}$ & 5 & 5 & 5 & 5 & 6 & 6 \\
$A_{2}$ & 10 & 10 & 9 & 9 & 9 & 10 \\
$A_{3}$ & 9 & 9 & 10 & 10 & 10 & 9 \\
$A_{4}$ & 7 & 8 & 8 & 8 & 8 & 8 \\
$A_{5}$ & 1 & 1 & 1 & 1 & 1 & 1 \\
$A_{6}$ & 11 & 11 & 12 & 11 & 11 & 11 \\
$A_{7}$ & 15 & 14 & 14 & 14 & 14 & 14 \\
$A_{8}$ & 8 & 7 & 6 & 6 & 5 & 5 \\
$A_{9}$ & 14 & 12 & 11 & 13 & 12 & 12 \\
$A_{10}$ & 12 & 13 & 13 & 12 & 13 & 13 \\
$A_{11}$ & 3 & 3 & 4 & 3 & 3 & 3 \\
$A_{12}$ & 13 & 15 & 15 & 15 & 15 & 15 \\
$A_{13}$ & 4 & 6 & 7 & 7 & 7 & 7 \\
$A_{14}$ & 2 & 2 & 2 & 2 & 2 & 2 \\
$A_{15}$ & 6 & 4 & 3 & 4 & 4 & 4 \\
\hline
\end{tabular}

\section{Concluding remarks}

In this paper we have analyzed the TODIM method and suggested a simplification of this model avoiding the calculation of the relative weight of the criteria with respect to a reference criterion. In this way, the function providing the dominance degree of each alternative over the others with respect to the different criteria is simpler than that given in the original model proposed by Gomes and Lima $[14,15]$. Moreover, we have illustrated with examples that this method is vulnerable to two paradoxes concerning the weights of the model. To avoid these paradoxes we have established two properties, called weight consistency and weight monotonicity, that prevent their occurrence. Furthermore, we have proposed a generalization of the TODIM method and established conditions under which this new procedure satisfies the previous properties. It is worth mentioning that both the original and the generalized TODIM method are based on pairwise comparisons and, therefore, they may suffer the well-known rank reversal phenomenon; that is, the relative 
ranking between two alternatives could be reversed when another alternative is added or deleted. ${ }^{17}$ Notice also that expression (7) provides a great flexibility to the DMs, since they only need to choose nondecreasing functions to guarantee the fulfillment of weight consistency and weight monotonicity. In fact, we have also shown that two of the most prominent multicriteria methods, the SAW and the PROMETHEE II methods (assuming for the latter certain hypotheses), can be obtained from the generalized TODIM method. In addition to these two methods, expression (9) is very interesting because it allows us to reflect the spirit of the subjective value function without modifying the weights of the criteria. Hence, the generalized TODIM method seems an appropriate procedure for solving multicriteria problems in future research.

\section{Acknowledgements}

This work is partially supported by the Spanish Ministry of Economy and Competitiveness (Project ECO2016-77900-P) and ERDF. The author is grateful to four anonymous referees for comments and suggestions that helped improve the paper.

\section{References}

[1] M. Behzadian, R.B. Kazemzadeh, A. Albadvi, M. Aghdasi, PROMETHEE: A comprehensive literature review on methodologies and applications, European Journal of Operational Research 200 (2010) 198-215.

[2] D. Black, The Theory of Committees and Elections, Springer, New York, 1958.

[3] D. Black, Partial justification of the Borda count, Public Choice 28 (1976) 1-15.

[4] P.R. Blavatskyy, Back to the St. Petersburg Paradox?, Management Science 51 (2005) 677-678.

[5] J.P. Brans, L'ingénierie de la décision: Elaboration d'instruments d'aide à la décision. La méthode PROMETHEE, in: R. Nadeau, M. Landry (Eds.), L'aide à la Décision: Nature, Instruments et Perspectives d'Avenir, Presses de l'Université Laval, Québec, 1982, pp. 183-213.

[6] J.P. Brans, Y. De Smet, PROMETHEE methods, in: S. Greco, M. Ehrgott, J.R. Figueira (Eds.), Multiple Criteria Decision Analysis: State of the Art Surveys, Springer, New York, 2016, second edition, pp. 187-219.

[7] J.P. Brans, P. Vincke, A preference ranking organisation method (The PROMETHEE method for multiple criteria decisionmaking), Management Science 31 (1985) 647-656.

[8] P. Coughlin, A direct characterization of Black's first borda count, Economics Letters 4 (1979) 131-133.

[9] W. De Keyser, P. Peeters, A note on the use of PROMETHEE multicriteria methods, European Journal of Operational Research 89 (1996) 457-461.

\footnotetext{
${ }^{17}$ The appearance of this problem in some multicriteria methods is well known (see, for instance, Wang and Luo [39], Verly and De Smet [36], and Maleki and Zahir [28]).
} 
[10] L. Diaz-Balteiro, J. González-Pachón, C. Romero, Measuring systems sustainability with multi-criteria methods: A critical review, European Journal of Operational Research 258 (2017) 607-616.

[11] Z.P. Fan, X. Zhang, F.D. Chen, Y. Liu, Extended TODIM method for hybrid multiple attribute decision making problems, Knowledge-Based Systems 42 (2013) 40-48.

[12] D.S. Felsenthal, Review of paradoxes afflicting procedures for electing a single candidate, in: D.S. Felsenthal, M. Machover (Eds.), Electoral Systems: Paradoxes, Assumptions, and Procedures, Studies in Choice and Welfare, Springer, Berlin, 2012, pp. 19-91.

[13] L.F.A.M. Gomes, X.I. González, Behavioral multi-criteria decision analysis: further elaborations on the TODIM method, Foundations of Computing and Decision Sciences 37 (2012) 3-8.

[14] L.F.A.M. Gomes, M.M.P.P. Lima, TODIM: Basics and application to multicriteria ranking of projects with environmental impacts, Foundations of Computing and Decision Sciences 16 (1991) 113-127.

[15] L.F.A.M. Gomes, M.M.P.P. Lima, From modelling individual preferences to multicriteria ranking of discrete alternatives: A look at prospect theory and the additive difference model, Foundations of Computing and Decision Sciences 17 (1992) 171-184.

[16] L.F.A.M. Gomes, M.A.S. Machado, L.A.D. Rangel, Behavioral multi-criteria decision analysis: the TODIM method with criteria interactions, Annals of Operations Research 211 (2013) 531-548.

[17] L.F.A.M. Gomes, L.A.D. Rangel, An application of the TODIM method to the multicriteria rental evaluation of residential properties, European Journal of Operational Research 193 (2009) 204-211.

[18] L.F.A.M. Gomes, L.A.D. Rangel, F.J.C. Maranhão, Multicriteria analysis of natural gas destination in Brazil: An application of the TODIM method, Mathematical and Computer Modelling 50 (2009) 92-100.

[19] K. Govindan, M. Kadziński, R. Sivakumar, Application of a novel PROMETHEE-based method for construction of a group compromise ranking to prioritization of green suppliers in food supply chain, Omega 71 (2017) 129-145.

[20] S. Greco, M. Ehrgott, J.R. Figueira (Eds.), Multiple Criteria Decision Analysis: State of the Art Surveys, volume 233 of International Series in Operations Research E Management Science, Springer, New York, second edition, 2016.

[21] C.L. Hwang, K. Yoon, Multiple Attribute Decision Making: Methods and Applications, Springer-Verlag, Berlin, 1981.

[22] A. Jahan, K.L. Edwards, A state-of-the-art survey on the influence of normalization techniques in ranking: Improving the materials selection process in engineering design, Materials and Design 65 (2015) 335-342.

[23] P. Ji, H.Y. Zhang, J.Q. Wang, Fuzzy decision-making framework for treatment selection based on the combined QUALIFLEX-TODIM method, International Journal of Systems Science 48 (2017) 3072-3086.

[24] P. Ji, H.Y. Zhang, J.Q. Wang, A projection-based TODIM method under multi-valued neutrosophic environments and its application in personnel selection, Neural Computing and Applications 29 (2018) 221-234.

[25] D. Kahneman, A. Tversky, Prospect theory: An analysis of decision under risk, Econometrica 47 (1979) $263-291$.

[26] Y.S. Lee, H.S. Shih, Incremental analysis for generalized TODIM, Central European Journal of Operations Research 24 (2016) 901-922.

[27] R. Lourenzutti, R.A. Krohling, A study of TODIM in a intuitionistic fuzzy and random environment, Expert Systems with Applications 40 (2013) 6459-6468.

[28] H. Maleki, S. Zahir, A comprehensive literature review of the rank reversal phenomenon in the Analytic Hierarchy Process, Journal of Multi-Criteria Decision Analysis 20 (2013) 141-155. 
[29] M. Marttunen, J. Lienert, V. Belton, Structuring problems for Multi-Criteria Decision Analysis in practice: A literature review of method combinations, European Journal of Operational Research 263 (2017) 1-17.

[30] E. Mulliner, N. Malys, V. Maliene, Comparative analysis of MCDM methods for the assessment of sustainable housing affordability, Omega 59 (2016) 146-156.

[31] A.C. Passos, L.F.A.M. Gomes, TODIM-FSE: A multicriteria classification method based on prospect theory, Multiple Criteria Decision Making 9 (2014) 123-139.

[32] A.C. Passos, M.G. Teixeira, K.C. Garcia, A.M. Cardoso, L.F.A.M. Gomes, Using the TODIM-FSE method as a decisionmaking support methodology for oil spill response, Computers \& Operations Research 42 (2014) 40-48.

[33] J. Qin, X. Liu, W. Pedrycz, An extended TODIM multi-criteria group decision making method for green supplier selection in interval type-2 fuzzy environment, European Journal of Operational Research 258 (2017) 626-638.

[34] N. Soni, R.A. Christian, N. Jariwala, Pollution potential ranking of industries using classical TODIM method, Journal of Environmental Protection 7 (2016) 1645-1656.

[35] A. Tversky, D. Kahneman, Advances in prospect theory: Cumulative representation of uncertainty, Journal of Risk and Uncertainty 5 (1992) 297-323.

[36] C. Verly, Y. De Smet, Some results about rank reversal instances in the PROMETHEE methods, International Journal of Multicriteria Decision Making 3 (2013) 325-345.

[37] J. Wang, J.Q. Wang, H.Y. Zhang, A likelihood-based TODIM approach based on multi-hesitant fuzzy linguistic information for evaluation in logistics outsourcing, Computers \& Industrial Engineering 99 (2016) 287-299.

[38] X. Wang, E. Triantaphyllou, Ranking irregularities when evaluating alternatives by using some ELECTRE methods, Omega 36 (2008) 45-63.

[39] Y.M. Wang, Y. Luo, On rank reversal in decision analysis, Mathematical and Computer Modelling 49 (2009) 1221-1229.

[40] S.M. Yu, J. Wang, J.Q. Wang, An extended TODIM approach with intuitionistic linguistic numbers, International Transactions in Operational Research 25 (2018) 781-805.

[41] X. Zhang, Z. Xu, The TODIM analysis approach based on novel measured functions under hesitant fuzzy environment, Knowledge-Based Systems 61 (2014) 48-58. 